\title{
EXISTENCE AND UNIQUENESS OF SOLUTIONS FOR SOME NEUTRAL DIFFERENTIAL EQUATIONS WITH STATE-DEPENDENT DELAYS
}

\author{
KHELIFA DAOUDI ${ }^{1}$, JOHNNY HENDERSON ${ }^{2}$, \\ AND ABDELGHNAI OUAHAB ${ }^{3}$ \\ ${ }^{1}$ Laboratory of Mathematics \\ Universoity Sidi Bel Abbes \\ P.O. Box 89, 22000 Sidi-Bel-Abbès, ALGERIA \\ ${ }^{2}$ Department of Mathematics \\ Baylor University \\ Waco, Texas, 76798-7328, USA
}

\begin{abstract}
In this work, we establish several results about the existence and uniqueness of solutions for some neutral differential equations with state-dependent delays. We assume that the linear part generates a strongly continuous semigroup on a general Banach space.
\end{abstract}

AMS Subject Classification: $34 \mathrm{G} 20,34 \mathrm{~K} 20,34 \mathrm{~K} 30$

Key Words: existence and uniqueness of solutions, neutral differential equations with state-dependent delays, Banach space, strongly continuous semigroup

Received: November 8, 2017; Accepted: April 12, 2018;

Published: May 10, $2018 \quad$ doi: $10.12732 /$ caa.v22i3.1

Dynamic Publishers, Inc., Acad. Publishers, Ltd. http://www.acadsol.eu/caa

\section{INTRODUCTION}

In this paper, we study the existence and uniqueness of solutions for neutral differential equations with state-dependent delays of the following form, 


$$
\begin{array}{r}
\frac{d}{d t}(x(t)-g(t, x(t-\eta(t))))=A(x(t)-g(t, x(t-\eta(t))))+f\left(t, x_{t}, x\left(t-\tau\left(t, x_{t}\right)\right)\right) \\
t \in[0, T], \quad
\end{array}
$$

with initial condition

$$
x(t)=\varphi(t), t \in[-r, 0],
$$

where A generates a strongly continuous semigroup $(S(t))_{t \geq 0}$ on a Banach space $E, f: J \times C([-r, 0], E) \times E \rightarrow E, g: J \times E \rightarrow E$ are given functions, and $\varphi:[-r, 0] \rightarrow E, \tau:[0, T] \times C([-r, 0], E) \rightarrow[0, r]$ and $\eta: J \rightarrow[0, r]$ are also given continuous functions.

For any function $x$ defined on $[-r, T]$ and any $t \in J$ we denote by $x_{t}$ the element of $C([-r, 0], E)$ defined by

$$
x_{t}(\theta)=x(t+\theta), \theta \in[-r, 0]
$$

Here $x_{t}(\cdot)$ represents the history of the state from time $t-r$, up to the present time $t$.

The theory of functional differential equations has emerged as an important branch of nonlinear analysis. It is worth mentioning that several important problems of the theory of ordinary and delay differential equations lead to investigations of functional differential equations of various types, see the books of Hale and Verduyn Lunel [11], Kolmanovskii and Myshkis [17], Wu [26], and the references therein.

Functional differential equation with state-dependent delays appear frequently in applications such as model equations (see, e.g., [3, 4, 6, 21]) and the study of such equations is an active research area (see, e.g., [7, 2, 9, 12 , $13,14,15,16,18,19,20,23,25]$.

Abstract neutral differential equations arise in many areas of applied mathematics. For this reason, they have largely been studied during the last few decades. The literature related to ordinary neutral differential equations is very extensive, for which we refer the reader to [11] only, which contains a comprehensive description of such equations.

This paper is organized as follows: in Section 2, we will recall briefly some basic definitions and preliminary facts which will be used throughout the following sections. In Section 3 we give one of our main existence results for solutions of (1.1)-(1.2), with the proof based on Banach's fixed point theorem. 
In Section 4, we give two other existence results for solutions of (1.1)-(1.2). Their proofs involve the measure of noncompactness paired in one result with a Mönch fixed point theorem and paired in the other result with a Darbo fixed point theorem.

\section{PRELIMINARIES}

In this section, we introduce notations, definitions, and preliminary facts which are used throughout this paper. Let $(E,\|\cdot\|)$ be a Banach space.

$C([-r, T], E)$ is the Banach space of all continuous functions from $[-r, T]$ into $E$ with the norm

$$
\|x\|_{\infty}=\sup _{\theta \in[-r, 0]} \sup _{t \in[0, T]}\|x(t+\theta)\| .
$$

$L^{1}([0, T], E)$ denotes the Banach space of measurable functions $x:[0, T] \rightarrow E$ which are Bochner integrable and is normed by

$$
\|x\|_{L^{1}}=\int_{0}^{T}\|x(t)\| d t .
$$

In a normed space $\left(X,\|\cdot\|_{X}\right)$, the open ball around a point $x_{0}$ with radius $R$ is denoted by $B_{X}\left(x_{0}, R\right)$, i.e., $B_{X}\left(x_{0}, R\right):=\left\{x \in X:\left\|x-x_{0}\right\|_{X}<R\right\}$, and the corresponding closed ball, by $\bar{B}_{X}\left(x_{0}, R\right)$.

Let $B(E)$ be the Banach space of bounded linear operators.

Definition 2.1. A one-parameter family $S(t)$ for of bounded linear operators on a Banach space $E$ is a $C_{0}$-semigroup (or strongly continuous) on $E$ if

(i) $S(t) \circ S(s)=S(t+s)$, for $t, s \geq 0$, (semigroup property),

(ii) $S(0)=I,($ the identity on $E)$;

(iii) the map $t \rightarrow S(t) x$ is strongly continuous, for each $x \in E$, i.e;

$$
\lim _{t \rightarrow 0} S(t)(x)=x, \forall x \in E .
$$

A semigroup of bounded linear operators $S(t)$, is uniformly continuous if

$$
\lim _{t \rightarrow 0}\|S(t)-I\|=0 .
$$

Here $I$ denotes the identity operator in $E$. 
Theorem 2.2. [24] If $S(t)$ is a $C_{0}$-semigroup, then there exist $\omega \geq 0$ and $M \geq 1$ such that

$$
\|S(t)\|_{B(E)} \leq M \exp (\beta t), \text { for } 0 \leq t<\infty
$$

Definition 2.3. Let $S(t)$ be a semigroup of class $\left(C_{0}\right)$ defined on $E$. The infinitesimal generator $A$ of $S(t)$ is the linear operator defined by

$$
A(x)=\lim _{h \rightarrow 0}=\frac{S(h)(x)-x}{h}, \text { for } x \in D(A),
$$

where $D(A)=\left\{x \in E \mid \lim _{h \rightarrow 0}=\frac{S(h)(x)-x}{h}\right.$ exists in $\left.E\right\}$.

Let us recall the following property:

Proposition 2.4. The infinitesimal generator $A$ is a closed, linear and densely defined operator in $E$. If $x \in D(A)$, then $S(t)(x)$ is a $C^{1}$-map and

$$
\frac{d}{d t} S(t)(x)=A(S(t)(x))=S(t)(A(x)) \text { on }[0, \infty) .
$$

Definition 2.5. ([5]) Let $E$ be a Banach space and $\Omega_{E}$ the family of bounded subsets of $E$. The Kuratowski measure of noncompactness is the map $\alpha$ : $\Omega_{E} \rightarrow[0, \infty)$ defined by

$$
\alpha(B)=\inf \left\{\epsilon>0: B \subseteq \cup_{i=1}^{n} B_{i} \text { and } \operatorname{diam}\left(B_{i}\right) \leq \epsilon\right\}
$$

Proposition 2.6. The Kuratowski measure of noncompactness satisfies the following properties (for more details see [5]).

(a) $\alpha(B)=0 \Leftrightarrow \bar{B}$ is compact ( $B$ is relatively compact).

(b) $\alpha(B)=\alpha(\bar{B})$.

(c) $A \subset B \Rightarrow \alpha(A) \leq \alpha(B)$.

(d) $\alpha(A+B) \leq \alpha(A)+\alpha(B)$.

(e) $\alpha(c B)=|c| \alpha(B) ; c \in \mathbb{R}$.

(f) $\alpha(\operatorname{conv} B)=\alpha(B)$. 


\section{UNIQUENESS OF MILD SOLUTIONS}

In this section we give our main existence result for problem (1.1)-(1.2). Before stating and proving this result, we give the definition of its mild solution.

Definition 3.1. We say that a continuous function $x:[-r, T] \rightarrow E$ is a mild solution of problem (1.1), (1.2) if $x(t)=\varphi(t), t \in[-r, 0]$ and

$$
\begin{aligned}
x(t)= & S(t)[\varphi(0)-g(0, x(-\eta(0)))]+g(t, x(t-\eta(t))) \\
& +\int_{0}^{t} S(t-s) f\left(s, x_{s}, x\left(s-\tau\left(s, x_{s}\right)\right)\right) d s, t \in J .
\end{aligned}
$$

Set $C:=C([-r, 0], E)$.

Lemma 3.2. (See [15]) Let $a>0, b \geq 0, r_{1}>0, r_{2} \geq 0, r=\max \left\{r_{1}, r_{2}\right\}$, and $v:[0, \sigma] \rightarrow[0, \infty)$ be continuous and nondecreasing. Let $u:[-r, \sigma] \rightarrow$ $[0, \infty)$ be continuous and satisfy the inequality

$$
u(t) \leq v(t)+b u\left(t-r_{1}\right)+a \int_{0}^{t} u\left(s-r_{2}\right) d s, t \in[0, \sigma] .
$$

Then $u(t) \leq d(t) e^{\text {ct }}$ for $t \in[0, \sigma]$, where $c$ is the unique positive solution of $c b e^{-c r_{1}}+a e^{-c r_{2}}=c$, and

$$
d(t)=\max \left\{\frac{v(t)}{1-b e^{-c r_{1}}}, \max _{-r \leq s \leq 0} e^{-c s} u(s)\right\}, t \in[0, \sigma]
$$

Let $\Omega_{1} \in C, \Omega_{2} \in E$ and $\Omega_{3} \in E$ be open subsets of their respective spaces. Let $T>0$ be finite, or $T=\infty$, in which case $[0, T]$ denotes the interval $[0, \infty)$.

We define the set

$$
\Pi=\left\{\varphi \in C: \varphi \in \Omega_{1}, \varphi(-\tau(0, \varphi)) \in \Omega_{2}, \varphi(-\eta(0)) \in \Omega_{3}\right\}
$$

Let us introduce the following hypotheses:

$\left(H_{1}\right) \quad A$ is the generator of a strongly continuous semigroup $S(t), t \in J$ which is compact for $t>0$ in the Banach space $E$. Let $M>0$ be such that

$$
\|S(t)\| \leq M \text { for all } t \in J
$$

$\left(H_{2}\right)$ (i) $f: J \times \Omega_{1} \times \Omega_{2} \rightarrow E$ is continuous; 
(ii) $f(t, \psi, u)$ is locally Lipschitz continuous in $\psi$ and $u$ in the following sense: for every finite $\sigma \in(0, T]$, for every closed and bounded subset $M_{1} \subset \Omega_{1}$ of $C$ and closed and bounded subset $M_{2} \subset \Omega_{2}$ of $E$, there exists a constant $L_{1}>0$ such that

$\left.\left.\| f\left(t, \psi_{1}, u_{1}\right)\right)-f\left(t, \psi_{2}, u_{2}\right)\right) \| \leq L_{1}\left(\sup _{\zeta \in\left[-r,-r_{0}\right]}\left\|\psi_{1}(\zeta)-\psi_{2}(\zeta)\right\|+\left\|u_{1}-u_{2}\right\|\right)$ for every $t \in[0, \sigma], \psi_{1}, \psi_{2} \in M_{1}$ and $u_{1}, u_{2} \in M_{2}$,

$\left(H_{3}\right)$ (i) $g: J \times \Omega_{3} \rightarrow E$ is continuous;

(ii) $g(t, u)$ is locally Lipschitz continuous in $u$ in the following sense: for every finite $\sigma \in(0, T]$, for every closed and bounded subset $M_{3} \subset \Omega_{3}$ of $E$, there exists a constant $0<L_{2}<1$ such that

$$
\left\|g\left(t, u_{1}\right)-g\left(t, u_{2}\right)\right\| \leq L_{2}\left\|u_{1}-u_{2}\right\|
$$

for every $t \in[0, \sigma]$ and $u_{1}, u_{2} \in M_{3}$,

$\left(H_{4}\right)$ there exists a constant $r_{0}>0$, such that

$$
r_{0} \leq \tau(t, \psi) \leq r, t \in[0, T], \text { and } \psi \in \Omega_{1}
$$

$\left(H_{5}\right)$ there exists a constant $L_{3}>0$, such that

$$
\|\varphi(\zeta)-\varphi(\bar{\zeta})\| \leq L_{3}\|\zeta-\bar{\zeta}\|
$$

for $\zeta, \bar{\zeta} \in[-r, 0]$.

Theorem 3.3. Assume that assumptions $\left(H_{1}\right)-\left(H_{4}\right)$ hold and let $\gamma \in \Pi$. Then, there exist $\delta>0$ and $0<\sigma \leq T$ finite numbers such that

(i) $P=\bar{B}_{C}(\gamma, \delta) \subset \Pi$;

(ii) the problem (1.1)-(1.2) has a unique mild solution on a maximal interval of existence $[-r, T)$ for all $\gamma \in P$.

Proof: $(i)$ Let $\gamma:=\widehat{\varphi} \in \Pi$. Since $\Omega_{1}, \Omega_{2}$ and $\Omega_{3}$ are open subsets of their respective spaces, there exists $\delta_{1}>0$ such that $B_{C}\left(\widehat{\varphi}, \delta_{1}\right) \subset \Omega_{1}$. Introduce the vectors $w_{1}=\widehat{\varphi}(-\tau(0, \widehat{\varphi}))$ and $w_{2}=\widehat{\varphi}(-\eta(0))$. Let $\varepsilon_{1}$ be such that $\bar{B}_{E}\left(w_{1}, \varepsilon_{1}\right) \subset \Omega_{2}$ and $\bar{B}_{E}\left(w_{2}, \varepsilon_{1}\right) \subset \Omega_{3}$.

The map 


$$
[0, T] \times C \rightarrow E, \quad(t, \psi) \mapsto \psi(-\tau(t, \psi))
$$

is continuous, since

$$
\begin{aligned}
\|\psi(-\tau(t, \psi))-\bar{\psi}(-\tau(\bar{t}, \bar{\psi}))\| \leq & \|\psi(-\tau(t, \psi))-\bar{\psi}(-\tau(t, \psi))\| \\
& +\|\bar{\psi}(-\tau(t, \psi))-\bar{\psi}(-\tau(\bar{t}, \bar{\psi}))\| \\
\leq & \|\psi-\bar{\psi}\|+\|\bar{\psi}(-\tau(t, \psi))-\bar{\psi}(-\tau(\bar{t}, \bar{\psi}))\| \\
\rightarrow & 0, \text { as } t \rightarrow \bar{t}, \psi \rightarrow \bar{\psi}
\end{aligned}
$$

Similarly, the map

$$
[0, T] \times C \rightarrow E, \quad(t, \psi) \mapsto \psi(-\eta(t))
$$

is also continuous; therefore, there exist $\delta_{2} \in\left(0, \delta_{1}\right]$ and $T_{1} \in(0, T]$ such that

$$
\begin{aligned}
& \left\|\psi(-\tau(t, \psi))-w_{1}\right\|<\varepsilon_{1},\left\|\psi(-\eta(t))-w_{2}\right\|<\varepsilon_{1} \\
& \qquad \text { for } t \in\left[0, T_{1}\right] \text { and } \psi \in B_{C}\left(\widehat{\varphi}, \delta_{2}\right) .
\end{aligned}
$$

In particular, we get that for $\varphi \in B_{C}\left(\widehat{\varphi}, \delta_{2}\right)$, it follows $\varphi \in \Omega_{1}, \varphi(-\tau(0, \varphi)) \in$ $\Omega_{2}, \varphi(-\eta(0)) \in \Omega_{3}$. Therefore, part $(i)$ of the theorem holds for any $0<\delta \leq$ $\delta_{2}$.

Fix $\varepsilon_{0}>0$. The continuity of the map $(t, \psi) \mapsto f(t, \psi, \psi(-\tau(t, \psi)))$ yields that there exist $\delta_{3} \in\left(0, \delta_{2}\right]$ and $T_{2} \in\left(0, T_{1}\right]$ such that

$$
\|f(t, \psi, \psi(-\tau(t, \psi)))-f(0, \varphi, \varphi(-\tau(t, \varphi)))\|<\varepsilon_{0}
$$

for $t \in\left[0, T_{2}\right], \psi \in B_{C}\left(\widehat{\varphi}, \delta_{3}\right)$.

Similarly,

$$
\|g(t, \psi(-\eta(t)))-g(0, \varphi(-\eta(0)))\|<\varepsilon_{0}
$$

for $t \in\left[0, T_{2}\right], \psi \in B_{C}\left(\widehat{\varphi}, \delta_{3}\right)$.

Define the sets

$$
M_{2}=\bar{B}_{E}\left(w_{1}, \varepsilon_{1}\right), \quad M_{3}=\bar{B}_{E}\left(w_{2}, \varepsilon_{1}\right) .
$$

The extension of the function $\psi \in C$ to the interval $[-r, \infty)$ by the constant value $\psi(0)$ will be denoted by

$$
\widetilde{\psi}(t)= \begin{cases}\psi(t), & \mathrm{t} \in[-r, 0] \\ \psi(0), & \mathrm{t} \geq 0\end{cases}
$$


We define the following constants

$$
\begin{aligned}
K_{1} & =\|f(0, \varphi, \varphi(-\tau(t, \varphi)))\|+\varepsilon_{0}, \\
K_{2} & =\|g(0, \varphi(-\eta(0)))\|+\varepsilon_{0}, \\
K_{3} & =\|\varphi(0)\|, \\
\beta & =\max \left\{(M+1) K_{3},(M+1) K_{2}, \sigma M K_{1}\right\}, \\
\delta & =\frac{\delta_{3}}{3}, \\
\sigma & =\min \left\{T_{2}, r_{0}\right\} .
\end{aligned}
$$

Then, let

$$
\max \left\{3 \beta, r L_{3}\right\} \leq \delta
$$

and

$$
\begin{aligned}
& E_{0}=\{u \in C([-r, \sigma], E), u(t)=\varphi(t) \text { if } t \in[-r, 0] \text { and } \\
& \left.\sup _{t \in[0, \sigma]}\|u(t)-\varphi(0)\| \leq \delta\right\} .
\end{aligned}
$$

It is clear that $E_{0}$ is a closed set of $C([-r, \sigma], E)$. For $u \in E_{0}, \varphi \in B_{C}(\widehat{\varphi}, \delta)$, $t \in[0, \sigma]$ and $\zeta \in[-r, 0]$, we have

$$
\begin{aligned}
\|u(t+\zeta)-\widehat{\varphi}(\zeta)\| & \leq\|u(t+\zeta)-\widetilde{\varphi}(t+\zeta)\|+\|\widetilde{\varphi}(t+\zeta)-\widetilde{\varphi}(\zeta)\| \\
& +\|\varphi(\zeta)-\widehat{\varphi}(\zeta)\| \\
& <\delta+r L_{3}+\delta \\
& \leq \delta+\delta+\delta \\
& \leq \delta_{3}
\end{aligned}
$$

and hence $\left\|u_{t}-\widehat{\varphi}\right\|_{C}<\delta_{3}$. Consequently, $u_{t} \in B_{C}\left(\widehat{\varphi}, \delta_{3}\right) \subset \Omega_{1}$, and so

$$
\begin{gathered}
\left\|f\left(t, u_{t}, u\left(t-\tau\left(t, u_{t}\right)\right)\right)\right\| \leq K_{1}, \\
\|g(t, u(t-\eta(t)))\| \leq K_{2},
\end{gathered}
$$

and $\psi=u_{t}$ satisfies $(3.1)$ for $u \in E_{0}, \varphi \in B_{C}(\widehat{\varphi}, \delta)$, and $t \in[0, \sigma]$. Therefore the definitions of $M_{2}, M_{3}$ and (3.1) yield

$$
u_{t}\left(-\tau\left(u_{t}\right)\right) \in M_{2}, u_{t}(-\eta(t)) \in M_{3}
$$


for $t \in[0, \sigma], u \in E_{0}$, and $\varphi \in B_{C}(\widehat{\varphi}, \delta)$. Transform the problem (1.1)-(1.2) into a fixed point problem. Consider the operator

$$
N: E_{0} \rightarrow C([-r, \sigma], E)
$$

defined by

$$
(N x)(t)= \begin{cases}\varphi(t), & \mathrm{t} \in[-r, 0] \\ S(t)[\varphi(0)-g(0, x(-\eta(0)))]+g(t, x(t-\eta(t))) & \\ +\int_{0}^{t} S(t-s) f\left(s, x_{s}, x\left(s-\tau\left(s, x_{s}\right)\right)\right) d s, & \mathrm{t} \in J .\end{cases}
$$

Note that a fixed point of $N$ is a mild solution of (1.1)-(1.2). We will show that

$$
N\left(E_{0}\right) \subseteq E_{0}
$$

Let $v \in E_{0}$ and $t \in[0, \sigma]$. We have

$$
\begin{aligned}
\|N(v)(t)-\varphi(0)\| \leq & \|S(t)[\varphi(0)-g(0, v(-\eta(0)))]-\varphi(0)\|+\|g(t, v(t-\eta(t)))\| \\
& +\left\|\int_{0}^{t} S(t-s) f\left(s, v_{s}, v\left(s-\tau\left(s, v_{s}\right)\right)\right) d s\right\| \\
\leq & (M+1)\|\varphi(0)\|+M\|g(0, v(-\eta(0)))\|+\|g(t, v(t-\eta(t)))\| \\
& +M\left\|\int_{0}^{t} f\left(s, v_{s}, v\left(s-\tau\left(s, v_{s}\right)\right)\right) d s\right\| \\
\leq & (M+1) K_{3}+M K_{2}+K_{2}+M K_{1} \int_{0}^{t} d s \\
\leq & (M+1) K_{3}+(M+1) K_{2}+M \sigma K_{1} \\
\leq & 3 \beta \leq \delta .
\end{aligned}
$$

Hence,

$$
N\left(E_{0}\right) \subseteq E_{0}
$$

On the other hand, let $v, w \in E_{0}$. Then for $t \in[0, \sigma]$, we have

$$
\begin{aligned}
\|N(v)(t)-N(w)(t)\| \leq & \|S(t)[g(0, v(-\eta(0)))-g(0, w(-\eta(0)))]\| \\
& +\|g(t, v(t-\eta(t)))-g(t, w(t-\eta(t)))\| \\
& +\| \int_{0}^{t} S(t-s)\left[f\left(s, v_{s}, v\left(s-\tau\left(s, v_{s}\right)\right)\right)\right.
\end{aligned}
$$




$$
\begin{aligned}
& \left.-f\left(s, w_{s}, w\left(s-\tau\left(s, w_{s}\right)\right)\right)\right] d s \| \\
\leq & M L_{2}\|v(-\eta(0))-w(-\eta(0))\| \\
& +L_{2}\|v(t-\eta(t))-w(t-\eta(t))\| \\
& +M L_{1} \int_{0}^{t} \sup _{\zeta \in\left[-r,-r_{0}\right]}\left\|v_{s}(\zeta)-w_{s}(\zeta)\right\| \\
& +M L_{1} \int_{0}^{t}\left\|v\left(s-\tau\left(s, v_{s}\right)\right)-w\left(s-\tau\left(s, w_{s}\right)\right)\right\| d s \\
\leq & M L_{2}\|v(-\eta(0))-w(-\eta(0))\| \\
& +L_{2}\|v(t-\eta(t))-w(t-\eta(t))\| \\
& +M L_{1} \int_{0}^{t} \sup _{\zeta \in[-r,-\sigma]}\left\|v_{s}(\zeta)-w_{s}(\zeta)\right\| \\
& +M L_{1} \int_{0}^{t}\left\|v\left(s-\tau\left(s, v_{s}\right)\right)-w\left(s-\tau\left(s, w_{s}\right)\right)\right\| d s .
\end{aligned}
$$

Since $u_{t}(\zeta)=u(t+\zeta)=\varphi(t+\zeta)=\varphi_{t}(\zeta)$ for $t \in[0, \sigma]$ and $\zeta \in[-r,-\sigma]$. We have $t-\tau\left(t, \varphi_{t}\right) \leq t-r_{0} \leq t-\sigma \leq 0$ for $t \in[0, \sigma]$, so $u_{t}\left(-\tau\left(t, \varphi_{t}\right)\right)=\varphi_{t}$ for $t \in[0, \sigma]$, and $v(-\eta(0))=w(-\eta(0))=\varphi(-\eta(0))$. Then

$$
\begin{aligned}
\|N(v)(t)-N(w)(t)\| & \leq M L_{2}\|\varphi(-\eta(0))-\varphi(-\eta(0))\| \\
& +L_{2}\|v(t-\eta(t))-w(t-\eta(t))\| \\
+M L_{1} \int_{0}^{t} & {\left[\left\|\varphi_{s}-\varphi_{s}\right\|+\left\|\varphi\left(s-\tau\left(s, \varphi_{s}\right)\right)-\varphi\left(s-\tau\left(s, \varphi_{s}\right)\right)\right\|\right] d s } \\
\leq & L_{2}\|v(t-\eta(t))-w(t-\eta(t))\| \\
\leq & L_{2} \sup _{\theta \in[-r, 0]} \sup _{t \in[0, \sigma]}\|v(t+\theta)-w(t+\theta)\| \\
& \leq L_{2}\|v-w\|_{\infty} .
\end{aligned}
$$

Consequently,

$$
\|N(v)-N(w)\|_{\infty} \leq L_{2}\|v-w\|_{\infty}
$$

Since $L_{2}<1, N$ is a contraction. By the Banach fixed point theorem [8] we conclude that $N$ has a unique fixed point in $E_{0}$ and the problem (1.1)-(1.2) has a unique mild solution on $[-r, \sigma]$.

Let $u(t)$ be the unique mild solution of problem (1.1)-(1.2) defined on its maximal interval of existence $[0, T), T>0$. Assume that $T<\infty$ and

$$
\lim _{t \rightarrow T^{-}}\|u(t)\|<\infty
$$


Then, there exists a constant $\rho>0$ such that $\|u(t)\| \leq \rho$, for $t \in[-r, T)$.

Note that $\left(H_{2}\right)$ and $\left(H_{3}\right)$ imply that

$$
\begin{gathered}
\|f(t, \psi, \psi(-\tau(t, \psi)))-f(0, \varphi, \varphi(-\tau(0, \varphi)))\| \leq \\
\quad L_{1}(\|\psi-\varphi\|+\| \psi(-\tau(t, \psi)) \\
-\varphi(-\tau(0, \varphi)) \|)
\end{gathered}
$$

for $t \in[0, \sigma], \psi \in \bar{B}_{C}(\widehat{\varphi}, \delta)$. Similarly,

$$
\|g(t, \psi(-\eta(t)))-g(0, \varphi(-\eta(0)))\| \leq L_{2}\|\psi(-\eta(t))-\varphi(-\eta(0))\|
$$

for $t \in[0, \sigma], \psi \in \bar{B}_{C}(\widehat{\varphi}, \delta)$.

We define the following constants

$$
\begin{gathered}
c_{1}=\|f(0, \varphi, \varphi(-\tau(0, \varphi)))\|+L_{1}(\|\varphi\|+\|\varphi(-\tau(0, \varphi))\|), \\
c_{2}=\|g(0, \varphi(-\eta(0)))\|+L_{2}\|\varphi(-\eta(0))\| .
\end{gathered}
$$

Let $t \in[0, T)$. We obtain

$$
\begin{aligned}
\|u(t)\| \leq & \|S(t)[\varphi(0)-g(0, u(-\eta(0)))]\| \\
& +\|g(t, u(t-\eta(t)))\| \\
& +\left\|\int_{0}^{t} S(s) f\left(s, u_{s}, u\left(s-\tau\left(s, u_{s}\right)\right)\right) d s\right\| \\
\leq & M[\|\varphi(0)\|+\|g(0, u(-\eta(0)))\|]+L_{2}\|u(t-\eta(t))\|+c_{2}+M c_{1} t \\
& +M L_{1} \int_{0}^{t}\left[\left\|u_{s}\right\|+\left\|u\left(s-\tau\left(s, u_{s}\right)\right)\right\|\right] d s \\
\leq & M[\|\varphi(0)\|+\|g(0, u(-\eta(0)))\|]+L_{2}\|u(t-\eta(t))\|+c_{2}+t M c_{1} \\
& + \text { tML } L_{1}\|u\|_{\infty}+M L_{1} \int_{0}^{t}\left\|u\left(s-\tau\left(s, u_{s}\right)\right)\right\| d s \\
\leq & v(t)+L_{2}\left\|u\left(t-r_{1}\right)\right\|+M L_{1} \int_{0}^{t}\left\|u\left(s-r_{2}\right)\right\| d s
\end{aligned}
$$

where $r_{1}=\eta, r_{2}=\tau$ and

$$
v(t)=M[\|\varphi(0)\|+\|g(0, u(-\eta(0)))\|]+c_{2}+t M c_{1}+t M L_{1}\|u\|_{\infty} .
$$

By Lemma 3.2, it follows that

$$
\|u(t)\| \leq d(t) e^{c t}
$$


for $t \in[0, T)$, where $c$ is the unique positive solution of $c L_{2} e^{-c r_{1}}+M L_{1} e^{-c r_{2}}=$ $c$, and

$$
d(t)=\max \left\{\frac{v(t)}{1-L_{2} e-c r_{1}}, \max _{-r \leq s \leq 0} e^{-c s} u(s),\right\}, t \in[0, T) .
$$

It follows that $\lim _{t \rightarrow T^{-}} u(t)$ exists. Consequently, $u(t)$ can be extended to $T$, which contradicts the maximality of $[0, T)$.

\section{EXISTENCE OF MILD SOLUTIONS}

In this section we apply a technique based on noncompactness measure assumption on the nonlinear term in proving an existence result for problem (1.1)-(1.2).

We introduce some additional hypotheses:

$\left(H_{5}\right)$ The function $f: J \times C \times E \rightarrow E$ is continuous.

$\left(H_{6}\right)$ (i) There exist constants $c_{1} \geq 0$ and $c_{2} \geq 0$ such that

$$
\|g(t, u)\| \leq c_{1}\|u\|+c_{2} \text {, a.e. } t \in J, u \in E
$$

(ii) the function $g$ is completely continuous and for any bounded set $B$ in $\Omega$, the set $\{t \rightarrow g(t, x(t-\eta(t))): \quad \mathrm{x} \in B\}$ is equicontinuous in $\Omega$.

$\left(H_{7}\right)$ There exist $c_{3}>0, p \in L^{1}\left(J, \mathbb{R}_{+}\right)$and a continuous nondecreasing function $\psi:[0, \infty) \rightarrow[0, \infty)$ such that

$$
\|f(t, u, v)\| \leq p(t) \psi(\|u\|)+c_{3}\|v\|, \text { for each } u \in C, v \in E \text { and } t \in J .
$$

$\left(H_{8}\right)$ For each bounded $B \subset E, B^{\prime} \subset E$ and $t \in J$ we have

$$
\alpha\left(f\left(t, B, B^{\prime}\right)\right) \leq p(t) \alpha(B)+c_{3} \alpha\left(B^{\prime}\right) .
$$

$\left(H_{9}\right)$ For each $t \in J$ and bounded $B \subset E$ we have

$$
\alpha(g(t, B)) \leq c_{1} \alpha(B) .
$$

$\left(H_{10}\right)$ There exists $q>0$ such that

$$
M\|\varphi\|_{\infty}+(M+1)\left[c_{1} q+c_{2}\right]+M\left[\|p\|_{L^{1}} \psi(q)+T c_{3} q\right] \leq q .
$$


Theorem 4.1. Assume that $\left(H_{1}\right),\left(H_{5}\right),\left(H_{6}\right),\left(H_{7}\right),\left(H_{8}\right),\left(H_{9}\right)$ and $\left(H_{10}\right)$ hold. Suppose that

$$
\left[c_{1}+M\left(c_{1}+\|p\|_{L^{1}}+c_{3} T\right)\right]<1
$$

Then the problem (1.1)-(1.2) has at least one mild solution on $[-r, T]$.

Proof: Transform the problem (1.1)-(1.2) into a fixed point problem. Consider the operator

$$
N: \Omega \rightarrow \Omega
$$

defined by

$$
(N x)(t)= \begin{cases}\varphi(t), & \mathrm{t} \in[-r, 0] \\ S(t)[\varphi(0)-g(0, x(-\eta(0)))]+g(t, x(t-\eta(t))) & \\ +\int_{0}^{t} S(t-s) f\left(s, x_{s}, x\left(s-\tau\left(s, x_{s}\right)\right)\right) d s, & \mathrm{t} \in J\end{cases}
$$

Note that a fixed point of $N$ is a mild solution of (1.1)-(1.2).

We will show that $N$ satisfies the assumptions of the Mönch fixed point theorem $[1,22]$.

Consider the set

$$
B_{q}=\left\{u \in \Omega:\|u\|_{\infty} \leq q\right\}
$$

where $q$ is the constant defined in $\left(H_{10}\right)$. Clearly, the subset $B_{q}$ is closed, bounded, and convex.

The proof will be given in several steps.

Step 1: $N$ is continuous.

Using $\left(H_{6}\right)$, it suffices to show that the operator $N_{1}: \Omega \rightarrow \Omega$ defined by

$$
N_{1}(x)(t)= \begin{cases}\varphi(t), & \mathrm{t} \in[-r, 0] \\ S(t) \varphi(0)+\int_{0}^{t} S(t-s) f\left(s, x_{s}, x\left(s-\tau\left(s, x_{s}\right)\right)\right) d s, & \mathrm{t} \in J,\end{cases}
$$

is continuous.

Let $\left\{u_{n}\right\}$ be a sequence such that $u_{n} \rightarrow u$ in $\Omega$. Then

$$
\begin{aligned}
\left\|N_{1}\left(u_{n}\right)(t)-N_{1}(u)(t)\right\| \leq & \| \int_{0}^{t} S(t-s)\left[f\left(s, u_{n s}, u_{n}\left(s-\tau\left(s, u_{n s}\right)\right)\right)\right. \\
& \left.-f\left(s, u_{s}, u\left(s-\tau\left(s, u_{s}\right)\right)\right)\right] d s \|
\end{aligned}
$$




$$
\begin{aligned}
\leq & M \int_{0}^{t} \| f\left(s, u_{n s}, u_{n}\left(s-\tau\left(s, u_{n s}\right)\right)\right) \\
& -f\left(s, u_{s}, u\left(s-\tau\left(s, u_{s}\right)\right)\right) \| d s \\
\leq & M \int_{0}^{t} \sup _{\theta \in[-r, 0]} \sup _{s \in[0, T]} \| f\left(s, u_{n s}, u_{n}(s+\theta)\right) \\
& -f\left(s, u_{s}, u(s+\theta)\right) \| d s \\
\leq & M T\left\|f\left(\cdot, u_{n .}, u_{n}(\cdot)\right)-f\left(\cdot, u_{.}, u(\cdot)\right)\right\|_{\infty} .
\end{aligned}
$$

Since $f$ is a continuous function, we have

$$
\left\|N_{1}\left(u_{n}\right)-N_{1}(u)\right\|_{\infty} \leq M T\left\|f\left(\cdot, u_{n .}, u_{n}(\cdot)\right)-f(\cdot, u, u(\cdot))\right\|_{\infty} \rightarrow 0 \text { as } n \rightarrow \infty .
$$

Thus $N_{1}$ is continuous.

Step 2: $N$ maps $B_{q}$ into itself.

For each $u \in B_{q}$, by $\left(H_{6}\right),\left(H_{7}\right)$ and $\left(H_{10}\right)$, we have for each $t \in[0, T]$

$$
\begin{aligned}
\|N(u)(t)\| \leq & \|S(t)[\varphi(0)-g(0, u(-\eta(0)))]\|+\|g(t, u(-\eta(0)))\| \\
& +\| \int_{0}^{t} S(t-s) f\left(s, f\left(s, u_{s}, u\left(s-\tau\left(s, u_{s}\right)\right)\right) d s \|\right. \\
\leq & M\|\varphi(0)\|+(M+1)\left(c_{1} q+c_{2}\right)+M\left[\psi(q) \int_{0}^{t} p(s) d s+c_{3} q \int_{0}^{t} d s\right] \\
\leq & M\|\varphi\|_{\infty}+(M+1)\left[c_{1} q+c_{2}\right]+M \psi(q)\|p\|_{L^{1}}+M T c_{3} q .
\end{aligned}
$$

Thus

$$
\|N(u)\|_{\infty} \leq M\|\varphi\|_{\infty}+(M+1)\left[c_{1} q+c_{2}\right]+M\left[\psi(q)\|p\|_{L^{1}}+T c_{3} q\right] \leq q
$$

Step 3: $N\left(B_{q}\right)$ is bounded and equicontinuous.

By Step 2, it is obvious that $N\left(B_{q}\right) \subset B_{q}$ is bounded. Using $\left(H_{6}\right)$, it suffices to show that the operator $N_{1}$ defined in (3.2) is equicontinuous.

Let $0<\tau_{1}, \tau_{2} \in J, \tau_{1}<\tau_{2}$ and $B_{q}$ be a bounded set of $\Omega$ as in Step 2. Let $u \in B_{q}$ then for each $t \in J$ we have

$$
\begin{aligned}
\left\|N_{1}(u)\left(\tau_{2}\right)-N_{1}(u)\left(\tau_{1}\right)\right\| \leq & \left\|S\left(\tau_{2}\right) \varphi(0)-S\left(\tau_{1}\right) \varphi(0)\right\| \\
& +\int_{0}^{\tau_{1}-\epsilon}\left\|S\left(\tau_{2}-s\right)-S\left(\tau_{1}-s\right)\right\|\left[p(s) \psi(q)+c_{3} q\right] d s \\
& +\int_{\tau_{1}}^{\tau_{1}-\epsilon}\left\|S\left(\tau_{2}-s\right)-S\left(\tau_{1}-s\right)\right\|\left[p(s) \psi(q)+c_{3} q\right] d s
\end{aligned}
$$




$$
+\int_{\tau_{1}}^{\tau_{2}}\left\|S\left(\tau_{2}-s\right)\right\|\left[p(s) \psi(q)+c_{3} q\right] d s .
$$

The right-hand side tends to zero as $\tau_{2}-\tau_{1} \rightarrow 0$, and $\epsilon$ sufficiently small, since $S(t)$ is a strongly continuous operator and the compactness of $S(t)$ for $t>0$ implies the continuity in the uniform operator topology.

Now let $V$ be a subset of $B_{q}$ such that $V \subset \overline{\operatorname{conv}}(N(V) \cup\{0\}) . \quad V$ is bounded and equicontinuous and therefore the function $t \rightarrow v(t)=\alpha(V(t))$ is continuous on $J$. By $\left(H_{8}\right),\left(H_{9}\right)$, and the properties of the measure $\alpha$ we have for each $t \in J$,

$$
\begin{aligned}
v(t) \leq & \alpha(N(V)(t) \cup\{0\}) \\
\leq & \alpha(N(V)(t)) \\
\leq & c_{1}[M \alpha(V(-\eta(0)))+\alpha(V(t-\eta(t)))] \\
& +M \int_{0}^{t}\left[p(s) \alpha\left(V_{s}\right)+c_{3} \alpha\left(V\left(s-\tau\left(s, V_{s}\right)\right)\right)\right] d s \\
\leq & c_{1}[M v(-\eta(0))+v(t-\eta(t))]+M \int_{0}^{t}\left[p(s) v_{s}+c_{3} v\left(s-\tau\left(s, V_{s}\right)\right)\right] d s \\
\leq & c_{1}(M+1)\|v\|_{\infty}+M\left[\|p\|_{L^{1}}\|v\|_{\infty}+c_{3} T\|v\|_{\infty}\right] \\
\leq & {\left[c_{1}+M\left(c_{1}+\|p\|_{L^{1}}+c_{3} T\right]\|v\|_{\infty} .\right.}
\end{aligned}
$$

Then

$$
\|v\|_{\infty}\left(1-\left[c_{1}+M\left(c_{1}+\|p\|_{L^{1}}+c_{3} T\right)\right]\right) \leq 0 .
$$

Since $\left[c_{1}+M\left(c_{1}+\|p\|_{L^{1}}+c_{3} T\right)\right]<1$ it follows that $v(t)=0$ for each $t \in J$, and then $V(t)$ is relatively compact in $E$. In view of the Ascoli-Arzelà theorem, $V$ is relatively compact in $B_{q}$. As a consequence of the Mönch fixed theorem $[1,22]$ we deduce that $N$ has a fixed point which is a mild solution of problem (1.1)-(1.2).

For the next theorem we replace the condition (4.1) by

$$
c_{1}(M+1)<1 \text {. }
$$

Now, consider the Kuratowski measure of noncompactness $\alpha_{C}$ defined on the family of bounded subsets of the space $C(J, E)$ by

$$
\alpha_{C}(H)=\sup _{\theta \in[-r, 0]} \sup _{t \in J} e^{-\tau L(t)} \alpha(H(t+\theta)),
$$

where $L(t)=\int_{0}^{t} \tilde{l}(s) d s, \quad \tilde{l}(t)=M\left(p(t)+c_{3}\right), \quad \tau>\frac{1}{1-c_{1}(M+1)}$.

Our next result is based on the Darbo fixed point theorem [10]. 
Theorem 4.2. Assume that $\left(H_{1}\right),\left(H_{5}\right),\left(H_{6}\right),\left(H_{8}\right),\left(H_{9}\right)$ and (4.4) are satisfied. Then the problem (1.1)-(1.2) has at least one mild solution on $[-r, T]$.

Proof: As in Theorem 4.1, we can prove that the operator $N: B_{q} \rightarrow B_{q}$ defined in that theorem is continuous and $N\left(B_{q}\right)$ is bounded.

Now we show that the operator $N: B_{q} \rightarrow B_{q}$ is a strict set contraction, i.e., there is a constant $0 \leq \lambda<1$ such that $\alpha(N(H)) \leq \lambda \alpha(H)$ for any $H \subset B_{q}$. In particular, we need to prove that there exists a constant $0 \leq \lambda<1$ such that $\alpha_{C}(N(H)) \leq \lambda \alpha_{C}(H)$ for any $H \subset B_{q}$. For each $t \in J$ we have

$$
\begin{aligned}
\alpha((N(H)(t)) \leq & c_{1}[M \alpha(H(-\eta(0)))+\alpha(H(t-\eta(t)))] \\
& +M \int_{0}^{t}\left[p(s) \alpha\left(H_{s}\right)+c_{3} \alpha\left(H\left(s-\tau\left(s, H_{s}\right)\right)\right)\right] d s \\
\leq & c_{1}[M \alpha(H(-\eta(0)))+\alpha(H(t-\eta(t)))] \\
& +M \int_{0}^{t} e^{\tau L(s)} e^{-\tau L(s)}\left[p(s) \alpha\left(H_{s}\right)+c_{3} \alpha\left(H\left(s-\tau\left(s, H_{s}\right)\right)\right)\right] d s \\
\leq & c_{1}[M \alpha(H(-\eta(0)))+\alpha(H(t-\eta(t)))] \\
& +M \sup _{\theta \in[-r, 0]} \sup _{s \in J} e^{-\tau L(s)} \alpha(H(s+\theta)) \int_{0}^{t} e^{\tau L(s)}\left[p(s)+c_{3}\right] d s \\
\leq & c_{1}[M \alpha(H(-\eta(0)))+\alpha(H(t-\eta(t)))]+\alpha_{C}(H) \int_{0}^{t} \tilde{l}(s) e^{\tau L(s)} d s \\
\leq & c_{1}[M \alpha(H(-\eta(0)))+\alpha(H(t-\eta(t)))]+\alpha_{C}(H) \int_{0}^{t}\left(\frac{e^{\tau L(s)}}{\tau}\right)^{\prime} d s \\
\leq & c_{1}[M \alpha(H(-\eta(0)))+\alpha(H(t-\eta(t)))]+\alpha_{C}(H) \frac{1}{\tau} e^{\tau L(t)} .
\end{aligned}
$$

Then

$$
\begin{aligned}
e^{-\tau L(t)} \alpha((N(H)(t)) & \leq c_{1} e^{-\tau L(t)}[M \alpha(H(-\eta(0)))+\alpha(H(t-\eta(t)))]+\alpha_{C}(H) \frac{1}{\tau} \\
& \leq c_{1}[M+1] \sup _{\theta \in[-r, 0]} \sup _{s \in J} e^{-\tau L(s)} \alpha(H(s+\theta))+\alpha_{C}(H) \frac{1}{\tau} .
\end{aligned}
$$

Consequently,

$$
\alpha_{C}(N H) \leq\left[c_{1}(M+1)+\frac{1}{\tau}\right] \alpha_{C}(H) .
$$

So, the operator $N$ is a set contraction. By the Darbo fixed point theorem [10] we deduce that $N$ has a fixed point which is a mild solution of problem (1.1)-(1.2). 


\section{REFERENCES}

[1] R. P. Agarwal, M. Meehan and D. O'Regan, Fixed Point Theory and Applications, Cambridge Tracts in Mathematics, 141. Cambridge University Press, Cambridge, 2001.

[2] M. Adimy and K. Ezzinbi, A class of linear partial neutral functionaldifferential equations with nondense domain, J. Differential Equations 147 (1998), 285-332.

[3] W. G. Aiello, H. I. Freedman and J. Wu, Analysis of a model representing stage-structured population growth with state-dependent time delay, SIAM J. Appl. Math. 52 (1992), 855-869.

[4] O. Arino, M. L. Hbid and R. Bravo de la Parra, A mathematical model of growth of population of Esh in the larval stage: density-dependence effects, Math. Biosci. 150 (1998) 1-20.

[5] J. Banas̀ and K. Goebel, Measures of Noncompactness in Banach Spaces, Marcel Dekker, New York, 1980.

[6] Y. Cao, J. Fan and T. C. Gard, The effect of state-dependent delay on a stage-structured population growth model, Nonlinear Anal. 19 (1992), 95-105.

[7] E. A. Dads and K. Ezzinbi, Boundedness and almost periodicity for some state-dependent delay differential equations, Electron. J. Differential Equations 2002, No. 67 (2002), pp. 1-13.

[8] J. Dugundji and A. Granas, Fixed Point Theory, Springer-Verlag, New York, 2003.

[9] K. Ezzinbi and X. Fu, Existence and regularity of solutions for some neutral partial differential equations with nonlocal conditions, Nonlinear Anal. 57 (2004), 1029-1041.

[10] D.J. Guo, V. Lakshmikantham and X. Liu, Nonlinear Integral Equations in Abstract Spaces, Kluwer Academic Publishers, Dordrecht, 1996. 
[11] J. K. Hale and S. Verduyn Lunel, Introduction to Functional-Differential Equations, Applied Mathematical Sciences, 99, Springer-Verlag, New York, 1993.

[12] F. Hartung, Linearized stability for a class of neutral functional differential equations with state-dependent delays. Nonlinear Anal. 69 (5-6) (2008), 1629-1643.

[13] F. Hartung, Differentiability of solutions with respect to parameters in neutral differential equations with state-dependent delays. J. Math. Anal. Appl. 324 (1) (2006), 504-524.

[14] F. Hartung, Linearized stability in periodic functional differential equations with state-dependent delays. J. Comput. Appl. Math. 174 (2) (2005), 201-211.

[15] F. Hartung, On differentiability of solutions with respect to parameters in neutral differential equations with state-dependent delays. Annali di Matematica 192 (2013) ,17-47.

[16] E. Hernández, A. Prokopczyk and L. Ladeira, A note on partial functional differential equations with state-dependent delay. Nonlinear Anal. Real World Applications 7 (2006), 510-519.

[17] V. Kolmanovskii, and A. Myshkis, Introduction to the Theory and Applications of Functional-Differential Equations, Kluwer Academic Publishers, Dordrecht, 1999.

[18] H.P. Krishnan, Existence of unstable manifolds for a certain class of delay differential equations, Electron. J. Differential Equations 32 (2002), 1-13.

[19] T. Krisztin and O. Arino, The 2-dimensional attractor of a differential equation with state-dependent delays, J. Dyn. Differential Equations 13 (2001), 453-522.

[20] T. Luzyanina and K. Engelborghs, D. Rose, Numerical bifurcation analysis of differential equations with state-dependent delays, Internat. J. Bifur. Chaos Appl. Sci. Eng. 11 (2001), 737-753. 
[21] J. M. Mahafy and J. Bélair and M. C. Mackey, Hematopoietic model with moving boundary condition and state-dependent delay: applications in erythropoesis, J. Theoret. Biol. 190 (1998) 135-146.

[22] H. Mönch, Boundary value problems for nonlinear ordinary differential equations of second order in Banach spaces, Nonlinear Anal. 4 (5) (1980), 985-999.

[23] A. V. Rezounenko and J. Wu, A non-local PDE model for population dynamics with state-selective delay: Local theory and global attractors, J. Comput. Appl. Math. 190 (1-2) (2006), 99-113.

[24] A. Pazy, Semigroups of Linear operators and Applications to Partial Differential Equations. New York (NY): Springer-Verlag; 1983.

[25] D. R. Willé and C. T. H. Baker, Stepsize control and continuity consistency for state-dependent delay-differential equations, J. Comput. Appl. Math. 53 (2) (1994), 163-170.

[26] J. Wu, Theory and Applications of Partial Functional Differential Equations, Springer-Verlag, New York, 1996. 
